\title{
PANCREAS DIVISUM AND CHOLEDOCHAL CYST
}

\author{
S. ARULPRAKASH, R. BALAMURALI, T. PUGAZHENDHI, S. JEEVAN KUMAR
}

\section{ABSTRACT}

Pancreas divisum (PD) is the most common congenital variant of the pancreas, affecting $5 \%$ to $14 \%$ of the population. The ventral duct only drains the ventral pancreas through the major papilla, whereas the majority of the pancreas drains via the dorsal duct through the minor papilla. We report the case of a 21 -year-old woman with recurrent acute pancreatitis who presented with the rare finding of choledochal cyst and pancreas divisum (PD). She underwent minor papilla sphincterotomy and pancreatic duct stenting. Comparable literature findings of PD and choledochal cyst are discussed with regard to the presented case.

Key words: Bile duct cysts, choledochal cyst, congenital variants, pancreas divisum, pancreatobiliary malunion

DOI: $10.4103 / 0019-5359.53166$

\section{INTRODUCTION}

Pancreas divisum, resulting from a fusion failure of the ventral and dorsal pancreatic buds and characterized by a dominant Santorini duct, is considered to be a predisposing factor for recurrent attacks of acute pancreatitis. Choledochal cyst disease is dilatation of the extrahepatic biliary tree or intrahepatic biliary tree or both. It is a potential premalignant condition, and therapy of choledochal cysts should be surgical. Association between pancreas divisum and pancreatobiliary malunion has been reported in literature. Very few reports of association between pancreas

Department of Digestive Health and Diseases (Government Peripheral Hospital), Kilpauk Medical College, Chennai, India

Correspondence:

Dr. Arulprakash S.

Plot No.: 119 A, First Main Road, Second Cross Street, Lakshmi Nagar Extension, Porur, Chennai - 600 116, India

E-mail: drarulaash@yahoo.co.in divisum and choledochal cyst are described in literature.

\section{CASE REPORT}

A 21-year-old woman was admitted to our hospital with recurrent episodes of postprandial right upper quadrant abdominal pain, nausea and vomiting. She had an uneventful pregnancy and had delivered 3 months back. On physical examination, she had mild tenderness in epigastric and left hypochondrium region. Findings of systemic examination were otherwise normal. The results of a complete blood count and the serum levels of glucose and electrolytes, as well as lipid profile and calcium level, were within the normal range, as were the results of renal function tests. Total bilirubin was $0.4 \mathrm{mg} \%$ (normal, 0.1-1 $\mathrm{mg} \%$ ); AST- $44 \mathrm{U} / \mathrm{L}$ (normal, <40 U/L); ALT- $58 \mathrm{U} / \mathrm{L}$ (normal, <40 U/L); and alkaline phosphatase103 IU (normal, 35-135 IU). Serum amylase (638 U) and serum lipase (762 U) were 
elevated. Ultrasonogram of abdomen showed edematous pancreas. CT scan abdomen showed bulky pancreas; a lobulated nonenhancing intra-pancreatic collection in head and neck of pancreas, measuring $4.4 \times 3.1$ $\mathrm{cm}$, with perilesional fat stranding. Upper Gastrointestinal endoscopy findings were unremarkable. Patient was treated as having mild acute pancreatitis, and she improved. While on follow-up after 6 months, she was admitted with similar episode, and then the pain was associated with hyperamylasemia (S. amylase, $1728 \mathrm{U}$ ). Magnetic resonance cholangio pancreatogram (MRCP) done during the episode showed type I choledochal cyst with pancreas divisum and resolving pancreatitis [Figures 1,2]. Endoscopic retrograde cholangio pancreatogram (ERCP) confirmation and endoscopic minor papilla stenting and sphincterotomy were done. Patient was symptom free on follow-up, and excision of the choledochal cyst was planned.

\section{DISCUSSION}

Pancreas divisum results from a failure of the

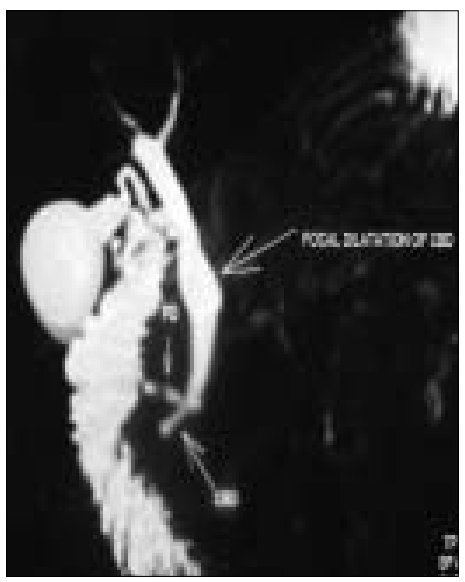

Figure 1: MRCP image showing pancreas divisum and choledochal cyst ducts of the embryological dorsal and ventral pancreas to fuse, the pancreatic exocrine secretion is drained preferentially via the Santorini duct and minor papilla, with only the uncinate process and part of the pancreatic body emptying through the duct of Wirsung and ampulla of Vater. Association of PD and recurrent pancreatitis may be attributed to a relative obstruction of the pancreatic juice flow through a relatively narrow minor papilla (dominant dorsal duct syndrome), causing increased intraductal pressure, duct distension, pain and pancreatitis. ${ }^{[1]}$ This hypothesis is indirectly supported by cessation of recurrent pancreatitis attacks after endoscopic treatment in some patients.

Variants of pancreatic ductal abnormalities include classical PD type I, with total failure of fusion; type II, with dorsal duct dominant drainage; and type III, incomplete divisum where a small communicating branch is present. ERCP and autopsy reports suggest an incidence of PD of approximately $7 \%$ (1\%14\%). Symptomatic (less than 5\%) PD patients develop acute, recurrent, idiopathic pancreatitis

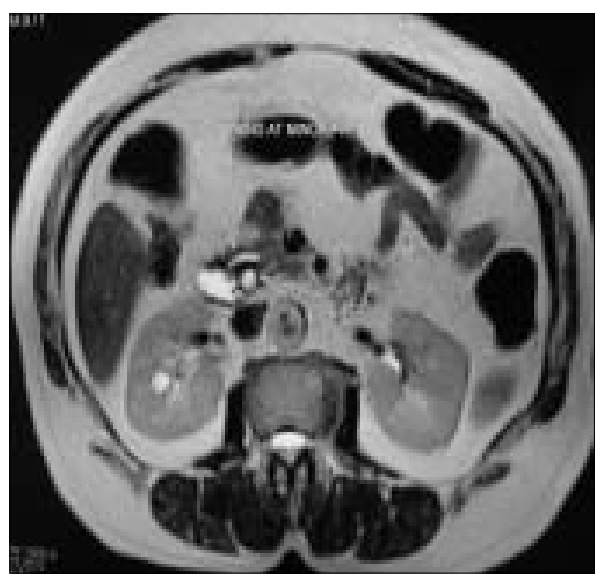

Figure 2: MRI abdomen showing choledochal cyst and accessory pancreatic duct draining into duodenum 
and postcholecystectomy syndrome. ${ }^{[1]}$

Choledochal cyst disease is dilatation of the extrahepatic biliary tree or intrahepatic biliary tree or both. Choledochal cysts are rare, occurring in approximately $1: 100,00-150,000$ live births. ${ }^{[2]}$ The majority (80\%) of these are diagnosed before 10 years of age. Choledochal cysts are generally classified into 5 types, using the Todani modification. ${ }^{[3]}$ The environment within a choledochal cyst is one of bile stasis, frequently resulting in the formation of stones and/or sludge, as well as biliary tract infection. The classic triad of right upper quadrant pain, mass and jaundice is seen more commonly in children. Adults may present with cholangitis or pancreatitis. The anatomic variant of a long common channel for the common bile and pancreatic ducts is known to be associated with choledochal cysts. It has been proposed that this anatomic variant promotes reflux of pancreatic juice into the common bile duct, resulting in inflammation, weakening of the bile duct wall and dilatation. ${ }^{[4]}$

Pancreatobiliary malunion is a congenital anomaly in which the junction of the pancreatic and biliary ducts located outside the duodenal wall, giving rise to a long common pancreatobiliary channel with a high risk of biliary tract carcinoma. ${ }^{[5,6]}$ Absence of a sphincter at the pancreatobiliary junction allows regurgitation of pancreatic juice and results in cystic lesions of bile duct. ${ }^{[4]} A$ similar hypothesis of a significant reflux of pancreatic juice to the bile duct can explain the association of choledochal cyst with pancreas divisum. Cystic bile duct anomalies require treatment due to 2 major reasons: first, the pain or recurrent episodes of cholangitis and pancreatitis; second, the malignant potency with a high risk of developing cystic adenocarcinoma of the bile duct. The type I cyst has the highest predominance of cancer, followed by the type IV cyst, with type III being the least common cyst harboring cancer. ${ }^{[7]}$ The management of patients with choledochal cysts consists of immediately treating any acute biliary tract process and planning an elective operative procedure. Surgery includes excision/ hepaticojejunostomy, ERCP sphincterotomy (type III cysts) and lobectomy (intrahepatic cysts) ${ }^{[8]}$

Due to paucity of reports, the exact incidence of association of PD with choledochal cyst is largely unknown. Clinically significant PD should be intervened by surgical sphincteroplasty or endoscopic methods (sphincterotomy, dilatation, stenting). Minor papilla endotherapy is most effective in patients with pancreas divisum and with recurrent acute pancreatitis with or without ductal changes, although patients with chronic pain and pancreas divisum respond poorly.

Bile duct resection, papillectomy, hepaticojejunostomy and jejunal reinsertion of the uncinate pancreatic duct in the same jejunal loop have been reported in literature. ${ }^{[9]}$ We planned a similar procedure for this patient. As she was a lactating mother, she decided to delay surgery by few months. Endoscopic minor papilla sphincterotomy was performed in this patient to prevent a recurrent episode of pancreatitis and discharged with advice of definite surgery.

To conclude, pancreas divisum with cystic bile duct lesions is a rare anatomic association. 
Significant reflux of pancreatic juice to the bile duct resulting in bile duct cyst can explain this association. Therapy should be a surgical resection of choledochal cyst under consideration of all particular individual anatomical features, as these bile duct anomalies represent precancerous lesions.

\section{REFERENCES}

1. Madura JA 2 nd , Madura JA. Diagnosis and management of sphincter of Oddi dysfunction and pancreas divisum. Surg Clin North Am 2007;87:1417-29.

2. Yamaguchi M. Congenital choledochal cyst: Analysis of 1,433 patients in the Japanese literature. Am J Surg 1980;140:653-7.

3. Todani T, Watanabe Y, Narusue M, Tabuchi K, Okajima K. Congenital bile duct cysts: Classification, operative procedures, and review of thirty-seven cases including cancer arising from choledochal cyst. Am J Surg 1977;134:263-9.

4. Petrasek J, Hucl T, Spicak J. Pancreaticobiliary malunion and incomplete pancreas divisum: An unusual cause of common bile duct obstruction. Adv Med Sci 2008;53:6-10.

5. Kamisawa T, Amemiya K, Tu Y, Egawa N, Sakaki $\mathrm{N}$, Tsuruta $\mathrm{K}$, et al. Clinical significance of a long common channel. Pancreatology 2002;2:122-8.

6. Jeong $\mathrm{IH}$, Jung $Y S$, Kim H, Kim BW, Kim JW, Hong $\mathrm{J}$, et al. Amylase level in extrahepatic bile duct in adult patients with choledochal cyst plus anomalous pancreatico-biliary ductal union. World J Gastroenterol 2005;11:1965-70.

7. Todani T, Tabuchi K, Watanabe Y. Carcinoma arising in the wall of congenital bile duct cysts. Cancer 1979;44:1134-41.

8. Weyant MJ, Maluccio MA, Bertagnolli MM, Daly JM. Choledochal cysts in adults: A report of two cases and review of the literature. Am J Gastroenterol 1998;93:2580-3.

9. Thilo H, Werner $H$, Jens W. Symptoms and surgical management of a distal choledochal cyst in a patient with pancreas divisum: Case report and review of the literature. Gastroenterology 2007;1:90-5.

Source of Support: Nil, Conflict of Interest: None declared.

\section{Staying in touch with the journal}

1) Table of Contents (TOC) email alert

Receive an email alert containing the TOC when a new complete issue of the journal is made available online. To register for TOC alerts go to $w \mathbf{w} w$.indianjmedsci.org/signup.asp.

\section{2) RSS feeds}

Really Simple Syndication (RSS) helps you to get alerts on new publication right on your desktop without going to the journal's w ebsite. You need a softw are (e.g. RSSReader, Feed Demon, FeedReader, My Yahoo!, New sGator and NewzCraw ler) to get advantage of this tool. RSS feeds can also be read through FireFox or M icrosoft Outlook 2007. Once any of these small (and mostly free) software is installed, add w w w .indianjmedsci.org/signup.asp as one of the feeds. 